\title{
Participation of nuclear localization signal 2 in the 3'-ETS domain of FLI1 in nuclear translocation of various chimeric EWS-FLI1 oncoproteins in Ewing tumor
}

\author{
NORIKAZU HONSEI ${ }^{1,2}$, TOGO IKUTA ${ }^{1}$, KATSUYOSHI KAWANA ${ }^{1}$, \\ YASUHIKO KANEKO $^{1}$ and KANAME KAWAJIRI ${ }^{1}$ \\ ${ }^{1}$ Research Institute for Clinical Oncology and ${ }^{2}$ Department of Orthopedics, \\ Saitama Cancer Center, Ina, Saitama 362-0806, Japan
}

Received February 10, 2006; Accepted April 18, 2006

\begin{abstract}
A $\mathrm{t}(11 ; 22)(\mathrm{q} 24 ; \mathrm{q} 12)$ translocation is present in $90 \%$ of Ewing's sarcoma, and results in the formation of the EWS-FLII fusion gene encoding an oncogenic transcription factor. To clarify the function of chimeric EWS-FLI1 proteins, an identification of a nuclear localization signal (NLS) in the EWS, FLI1 and EWS-FLI1 proteins is important because the chimeric oncoprotein may lose or gain NLS function different from native proteins resulting in different subcellular localization, and in deregulated gene expression. Furthermore, some studies reported that patients with one type of fusion gene ('type 1') had better overall survival than those with other types, suggesting that functional differences may be present among various fusion proteins. There has been only one study reporting a NLS in EWS, but none reporting those in FLI1 and EWS-FLI1. To clarify the molecular mechanisms of Ewing tumor development, we first identified the NLSs of EWS and FLI1. We allocated the NLS to amino acid residues 632-656 near the C-terminal region of EWS that is different from the previous study, and identified two NLSs of FLI1, NLS1 (63-90) in the N-terminal domain and NLS2 (319-360) in the 3 -ETS domain. In addition, the present study showed that all of the EWS-FLI1 fusion proteins completely reside in the nucleus without affecting the frequency of nuclear localization among variants, suggesting that NLS2 of FLI1 was used for nuclear translocation of various EWS-FLI1 fusion proteins.
\end{abstract}

\section{Introduction}

Ewing tumor is a primitive neuroectodermal tumor arising in bone or soft tissue of children and young adults. A $\mathrm{t}(11 ; 22)(\mathrm{q} 24 ; \mathrm{q} 12)$ translocation is present in $\leq 90 \%$ of patients

Correspondence to: Dr Yasuhiko Kaneko, Division of Cancer Diagnosis, Research Institute for Clinical Oncology, Saitama Cancer Center, Ina, Saitama 362-0806, Japan

E-mail:kaneko@cancer-c.pref.saitama.jp

Key words: EWS, FLI1, EWS-FLI1, nuclear localization signal, Ewing tumor with Ewing's sarcoma and results in the formation of the EWS-FLI1 fusion gene which encodes an oncogenic chimeric transcription factor (1-3). EWS belongs to the TET (TLS/FUS, EWS and TAFII68) family of proteins, which may act as adaptors between transcription and mRNA processing by interacting with components of the transcription apparatus and splicing factors (1-3). In contrast, FLI1 encodes a member of the ETS family of transcription factors having highly restricted tissue expression profiles, and appears to be involved in early hematopoietic, vascular, and neuroectodermal development $(4,5)$. The expression level of the chimeric EWS-FLI1 protein through gene rearrangement was directed by the EWS promoter, and a potent transactivation activity and different target genes of the EWS-FLI1 compared with that of native FLI1 were reported (6-9). Thus, the oncogenicity of the EWSFLI1 fusion transcription factor may depend on the deregulated activation of both FLI1 target genes in addition to some genes whose expression is not normally regulated by the FLI1 (6-9).

Since the $\mathrm{t}(11 ; 22)$ chromosome translocation breakpoints are distributed in introns 7-10 of the EWS gene and in introns 3-9 of the FLII gene, various chimeric genes are formed in Ewing tumors; i.e. type 1 (exon 7 of EWS/exon 6 of FLII), type 2 (exon 7 of EWS/exon 5 of FLII) (10). Some studies reported that patients with the 'type 1' fusion in the tumor had better overall survival than those with other fusion types $(11,12)$, although one study did not find such an association (13).

It is essential to study the nucleo-cytoplasmic trafficking of the chimeric transcription factor through gene rearrangements for elucidation of developing tumors, since the chimeric oncoprotein may lose or gain a nuclear localization signal (NLS) function different from a native transcription factor resulting in deregulated gene expression. Subcellular localization studies suggested that EWS protein is primarily nuclear but also cytoplasmic and on cell surface, and can shuttle between the nucleus and cytoplasm or cell surface under different physiologic conditions $(14,15)$. In contrast, FLI1 and EWSFLI1 proteins were localized in the nucleus (16). There has been only one study reporting a NLS in EWS (17), and none reporting NLSs in FLI1 and EWS-FLI1. To clarify the mechanism of nuclear-cytoplasmic transportation of EWS, 
FLI1 and EWS-FLI1 proteins, and the localization of the different chimeric proteins in the cells, we conducted experiments to determine the locations of NLSs of EWS, FLI1 and various EWS-FLI1 proteins. In contrast to a previous study (17), we identified a NLS of EWS in the C terminal region of the protein. Furthermore, we showed that FLI1 possesses two NLSs, one of which is located in the 3'ETS domain (4) and used for the cytoplasmic-nuclear transport of various EWS-FLI1 proteins, and all of the EWSFLI1 fusion proteins completely reside in the nucleus using NLS2 of FLI1.

\section{Materials and methods}

Cell culture. HeLa cells were maintained in Dulbecco's modified Eagle's medium supplemented with $10 \%$ fetal calf serum at $37^{\circ} \mathrm{C}$ with $5 \% \mathrm{CO}_{2}$ atmosphere.

Plasmid construction. Human EWS (1-656) or FLI1 (1-452) cDNA was prepared by polymerase chain reaction of human liver or universal Quick-Clone ${ }^{\mathrm{TM}}$ cDNA (Clontech Co., Palo Alto, CA) using specific primers and LA-Taq polymerase (Takara, Tokyo, Japan). Full-length cDNAs and various cDNA segments produced by polymerase chain reaction (PCR) were subcloned into adequate vectors such as pCMX. A BglII site in the coding sequence of EWS was deleted using the Quick Change $^{\mathrm{TM}}$ site-directed mutagenesis kit (Stratagene, La Jolla, CA) without changing the amino acid residue according to the manufacturer's instructions. A modified pSV- $\beta$ galactosidase ( $(-\mathrm{Gal})$ (18) vector and SRHis expression vector (19) were used to yield the in-frame fusion genes. All the constructs produced were confirmed by sequencing.

Construction of chimeric EWS-FLII fusion genes linked to His-Tag. EWS (1-264) was amplified by means of PCR using the His/EWS (1-656) vector as a template and specific primers to generate artificial $B a m \mathrm{HI}$ and $B g / I I$ sites at the $5^{\prime}$ and 3' ends, respectively. After double digestion with BamHI and $B g l I I$, the fragment was ligated to the $B g l$ II site of SRHis plasmid to generate His/EWS (1-264) vector. Various portions of FLI1 were amplified by PCR using His/FLI1 (1-452) vector as a template and specific primers to generate artificial $B g l$ II sites at both ends. After cleavage of amplified fragments of FLI1 with $B g l I I$, they were ligated into the $B g l I I$ site of His/EWS (1-264) vector to generate His-tagged chimeric EWS (1-264)-FLII fusions.

DNA transfection, Western blotting, and immunofluorescence. Transfection by electroporation and in situ staining of expressed of $\beta-$ Gal fusion proteins were performed as described previously (18). HeLa cells were also transfected with His/ EWS, His/FLI1, or His/EWS-FLI1 fusion expression plasmid by the lipofection method (19). The cells transfected were lysed in electrophoresis sample buffer $48 \mathrm{~h}$ after transfection and run on $10 \%$ sodium dodecyl sulfate-polyacrylamide gel electro-phoresis (SDS-PAGE). The proteins separated in the PAGE were transferred to a nitrocellulose membrane and probed with anti-6 x [His] rabbit IgG (Santa Cruz, CA) and developed using anti-rabbit IgG coupled to alkaline phosphatase. Immunocytochemical analysis was performed as
A

B
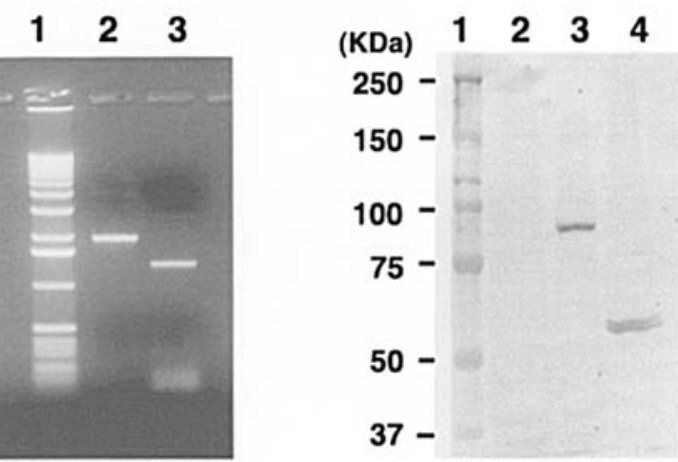

C
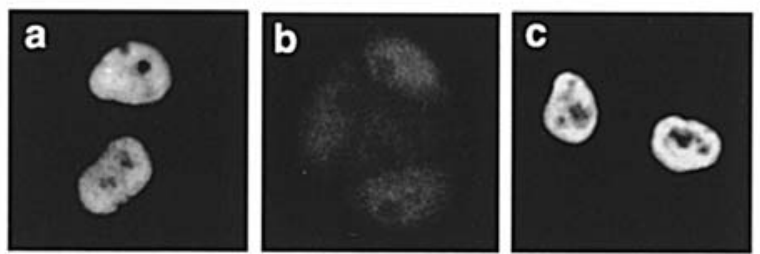

D
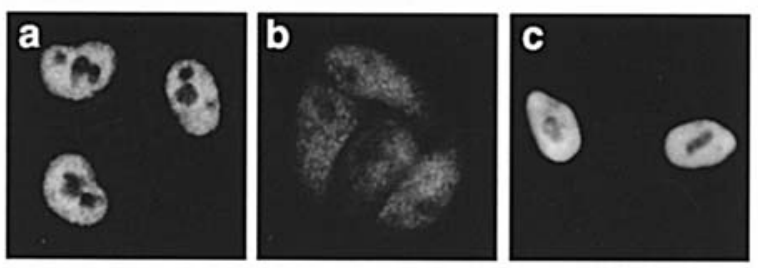

Figure 1. Transient expression of full-length EWS or FLI1 in HeLa cells. A, PCR amplification of human EWS (lane 2) or FLI1 (lane 3) using LA-Taq polymerase as described in Materials and methods. Lane 1 is a size marker of the 1-kb DNA ladder. B, Western blot analysis of expressed EWS or FLI1 fused with His-tag in HeLa cells. Immunoblotting was carried out using anti-6 x [His] rabbit IgG. His/EWS and His/FLI1 are shown in lanes 3 and 4, respectively. Size marker and negative control are shown in lanes 1 and 2, respectively. C, subcellular localization of expressed EWS in HeLa cells. HeLa cells were transfected with pCMX/EWS ( $a$ and $b$ ) or with SRHis/EWS (c) expression vector as described in Materials and methods. After fixation with $4 \%$ formaldehyde, cells on the coverslips were incubated with antiEWS goat antibody (a), anti-goat IgG antibody (b), or with anti-6 x [His] rabbit antibody (c) as the primary antibodies and fluorescein isothiocyanate (FITC)-conjugated anti-goat IgG (a and b) or anti-rabbit IgG (c) as the secondary antibodies. D, subcellular localization of expressed FLI1 in HeLa cells. HeLa cells were transfected with pCMX/FLI1 (a and b) or with SRHis/FLI1 (c) expression vector, and incubated with anti-FLI1 rabbit antibody (a), anti-rabbit IgG antibody (b), or with anti-6 x [His] rabbit antibody (c) as the primary antibodies and FITC-conjugated anti-rabbit $\operatorname{IgG}$ (a-c) as the secondary antibodies.

previously described (19), and cells were incubated with anti$6 \mathrm{x}$ [His] rabbit IgG followed by anti-rabbit IgG coupled with fluorescein isothio-cyanate (FITC). Immunohistochemical analysis of native EWS (pCMX/EWS) or FLI1 (pCMX/FLI1) expressed in HeLa cells was also carried out in the same manner using anti-EWS or anti-FLI1 antibodies (Santa Cruz).

Preparation and microinjection of GST-NLS-GFP fusion proteins. The candidates of NLS for EWS or FLII were amplified by means of PCR using the His/EWS (1-656) or His/FLI1 (1-452) vector as a template and specific primers to 


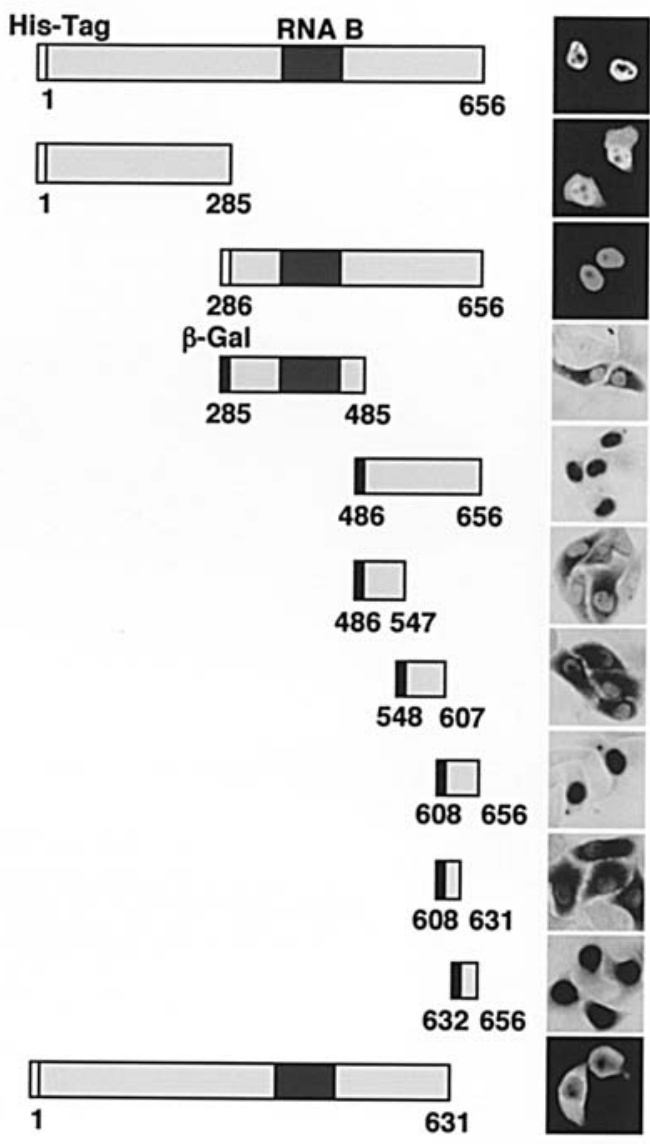

B

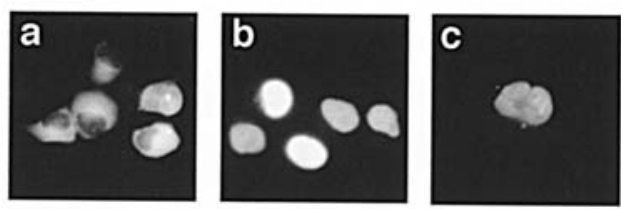

Figure 2. Identification of the region responsible for the nuclear localization of EWS. A, various EWS segments were synthesized using PCR, and the resulting fragments were fused to the modified $B-G a l$ control vector or SRHis plasmid as described in Materials and methods. B, microinjection of recombinant GST-EWS (632-656)-GFP protein into the cytoplasm of HeLa cells. After injection, the cells were fixed and the localization of the microinjected protein was examined by fluorescent microscopy. a, GST-GFP; b, GST-NLSc-GFP having an NLS of SV40 large T antigen. Amino acid sequence of NLSc used was PKKKRKV (18), and c, GST-EWS (632-656)GFP.

generate artificial $B g l$ II sites at both ends. After digestion with $B g I I I$, the fragment was ligated to the BamHI site of the glutathione S-transferase (GST)-green fluorescent protein (GFP) 2 vector (18) to produce an in-frame fusion gene. The GST-NLS-GFP vector was introduced into the Escherichia coli strain BL21, and purification of the expressed fusion protein was carried out as described previously (18). The purified preparations of fusion proteins were microinjected into the cytoplasm of HeLa cells, and the cells were incubated at $37^{\circ} \mathrm{C}$ for $30 \mathrm{~min}$ before fixation with $3.7 \%$ formaldehyde. The localization of injected GST-NLS-GFP fusion proteins was visualized under a Leica DMR microscope (Leica, Wetzlar, Germany).

\section{Results and Discussion}

Expression of EWS and FLII proteins in HeLa cells. The fulllength of human EWS (lane 2) or FLII (lane 3) cDNA was amplified by LA-PCR as shown in Fig. 1A, and subcloned into pCMX or SRHis A plasmid to yield their native or in-frame fusion genes. After sequencing followed by transfection, an adequate molecular size of the expressed fusion proteins of EWS (lane 3) or FLI1 (lane 4) was observed by Western blotting with anti-6 x [His] antibody, as shown in Fig. $1 \mathrm{~B}$. Transiently expressed native EWS (Fig. 1C) was observed in the nucleus by immunohistochemical staining with antibody to EWS (a), and nuclear localization was also confirmed when a fusion protein containing EWS linked to His-Tag at its $\mathrm{N}$-terminus was expressed and stained with anti- $6 \mathrm{x}$ [His] antibody (c). Nuclear localization of the native (a) or Histagged (c) FLI1 was also observed as shown in Fig. 1D. These results clearly showed that overexpressed EWS or FLI1 was localized in the nucleus. The lack of EWS in the cytoplasm in the present study may be caused by the different condition used in the present study and a small amount of EWS in the cytoplasm as suggested by a previous study (16).

Identification of NLS of EWS by transient expression assay. In a transfection assay of EWS and EWS-WT1, which is a chimeric oncoprotein associated with desmoplastic small round cell tumor $(20)$ caused by the $\mathrm{t}(11 ; 22)(\mathrm{p} 13 ; \mathrm{q} 12)$ translocation, Kim et al (17) showed the existence of a functional NLS in the N-terminal domain (NTD) of EWS (1-285), although there are only three basic amino acid residues in the NTD. If this is correct, the NLS of EWS is a novel one, different from the classical NLS composed of a basic amino acid cluster $(21,22)$, and comprised of non-basic amino acid segments.

To identify the NLS of EWS, we used two fusion proteins with the N-terminus of EWS linked to His-Tag or B-galactosidase (B-Gal). As shown in Fig. 2A, we divided EWS into two portions, NTD (1-285) and the C-terminal domain (CTD, 286-656), and investigated their potential for nuclear localization. In contrast to the previous findings of Kim et al (17), a fusion of NTD of EWS linked to His-Tag did not show any nuclear import activity at all, and a fusion of CTD of EWS did show clear nuclear import activity. Using a series of experiments linked to $\beta-\mathrm{Gal}$, we finally located the NLS of EWS at the C-terminal region between the 632nd and 656th amino acid residues. This result was supported by an expression study of a deletion mutant of EWS (1-631) fused with His-Tag, which had no nuclear import activity.

To confirm the ability of EWS (632-656) to translocate to the nucleus, we next examined the fate of purified recombinant protein fused with GST-GFP microinjected into the cytoplasm of HeLa cells (Fig. 2B). When microinjected into the cytoplasm of HeLa cells, the GST-GFP protein was localized to the cytoplasm even after incubation for $2 \mathrm{~h}$ (Fig. 2B-a). Microinjection of a fusion protein containing the SV40 NLSc (18) into the junction of the GST-GFP (GST-NLSc-GFP) revealed 
A
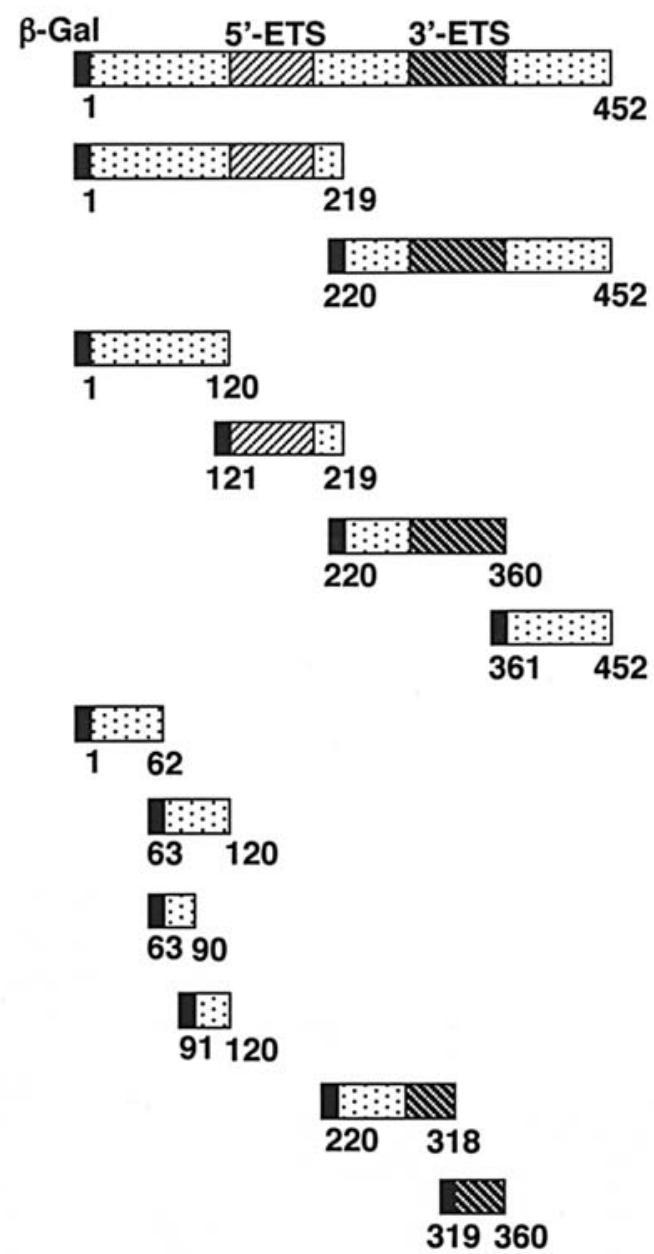

B
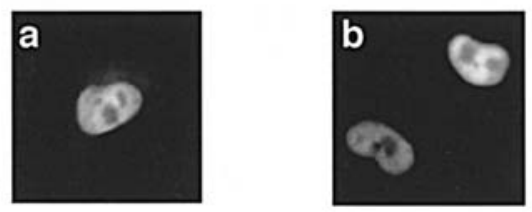

Figure 3. Identification of the region responsible for the nuclear localization of FLI1. A, various FLII segments were synthesized using PCR, and the resulting fragments were fused to the modified $B-G a l$ control vector. B, microinjection of recombinant GST-FLI1 (63-90)-GFP (a) or GST-FLI1 (319-360)-GFP (b) protein into the cytoplasm of HeLa cells. After injection followed by $30 \mathrm{~min}$ incubation, the cells were fixed and the localization of the microinjected protein was examined by fluorescent microscopy.

the efficient nuclear import within 30 min of incubation at $37^{\circ} \mathrm{C}$ (Fig. 2B-b). As was seen for the transient expression of B-Gal fusions (Fig. 2A), the GST-GFP fusion protein which contained EWS (632-656) showed efficient nuclear import activity, confirming that this region served as NLS (Fig. 2B-c). Thus, the present results strongly suggest that NLS of EWS exists near the C-terminal region of the EWS, constituting with basic amino acid clusters of the bipartite type, but not in the NTD as previously reported (17).

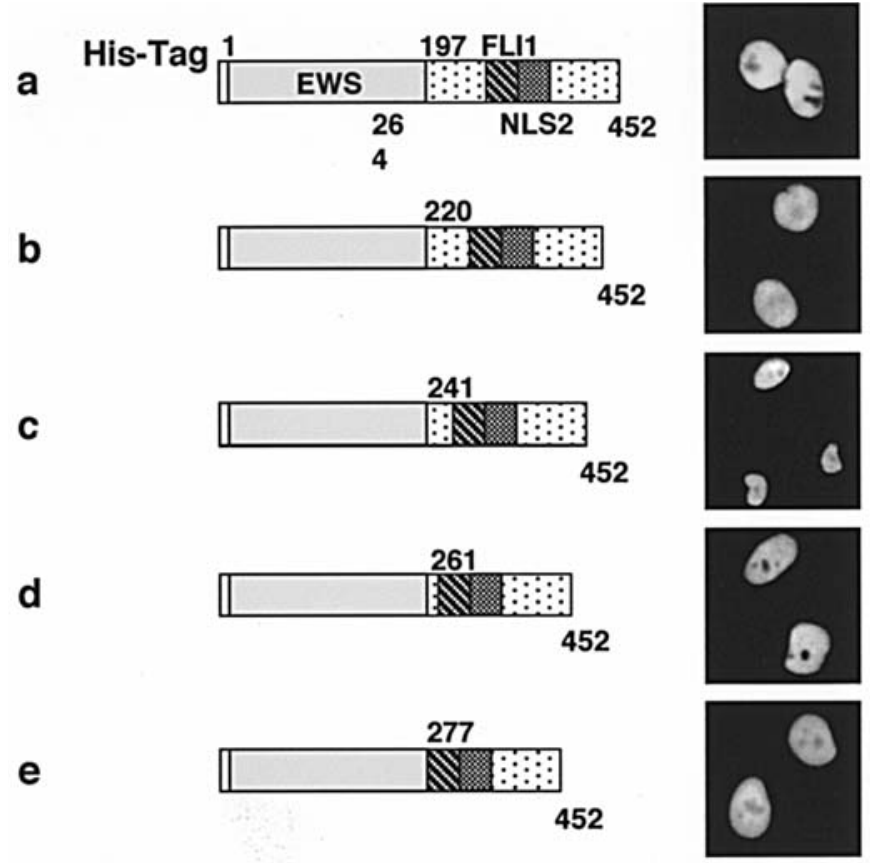

Figure 4. NLS2 of FLI1-dependent nuclear import of EWS-FLI1 chimeric oncoproteins. Variants of chimeric EWS-FLI1 proteins fused with His-Tag were generated and expressed in HeLa cells as described in Materials and methods. Subcellular localization was analyzed as described in Fig. 1. EWS-FLI1 fusions of the first exon 7 of EWS joined to exon 5 (a), exon 6 (b, type 1), exon 7 (c), exon 8 (d), and exon 9 (e) of FLII.

Two nuclear localization signals in FLI1 protein. There has been no study reporting the localization of NLSs in FLI1. As shown in Fig. 3A, nuclear accumulation activity of fulllength FLI1 linked to ß-Gal was clearly observed. To identify the region of FLI1 required for nuclear localization, various portions of cDNA for FLI1 were synthesized by PCR and ligated into the modified $B-G a l$ vector to produce fusion proteins. We first divided FLI1 into two portions, NTD (1-219) and CTD (220-452), and found that both of them showed nuclear import activity. Using a series of experiments, we finally located the two NLSs of FLI1 in the region between the 63rd and 90th (NLS1) and between the 319th and 360th (NLS2). In addition, microinjected purified fusion proteins of NLS1 or NLS2 linked to GST-GFP showed clear nuclear translocation activity within $30 \mathrm{~min}$ of incubation at $37^{\circ} \mathrm{C}$ (Fig. 3B), suggesting that the two regions serve as functional NLSs. In addition, the mapped NLS2 was overlapped completely with 3'-ETS DNA-binding domain of FLI1 that is conserved among various ETS families of transcription factors $(4,5,23)$. In contrast, NLS1 was not overlapped with 5'-ETS domain of FLI1 (121-194) (4).

Nuclear translocation of chimeric EWS-FLII proteins using NLS2 of FLI1. Fusion types of EWS-FLI1 protein were known to have prognostic significance in Ewing tumors $(11,12)$. Several investigators performed experiments to explain the cause of different outcomes of patients with type 1 fusion and those with non-type 1 fusions. Lin et al (24) found that transactivation of the type 1 protein was less effective than that of the non-type 1 proteins in Ewing and non-Ewing tumor cell lines. de Alava et al (25) examined Ewing tumors with 
type 1 or non-type 1 fusions by immunohistochemistry using Ki-67 and IGF-1R antibodies, and found higher Ki-67 proliferative index in non-type 1 tumors. A microarray study found few qualitative differences on gene expression between Ewing tumor cell lines with the type 1 fusion and those with the non-type 1 fusions (26). To verify whether various EWS-FLI1 chimeric proteins are uniformly translocated to nuclei, several chimeric fusion proteins of EWSFLI1 linked to His-Tag were produced, and subcellular localization of these expressed proteins was examined in HeLa cells. As shown in Fig. 4, all of the chimeric fusion proteins, including type 1 (b) and non-type 1(a, c-e) of EWSFLI1 fusion proteins, were completely localized in the nucleus in the same manner. Thus, it is likely that all types of EWS-FLI1 fusion proteins should be translocated into the nucleus using NLS2 in the 3'-ETS domain of FLI1, and that the subcellular localization of various chimeric proteins may not be related to the outcome.

In summary, we have identified the NLS of EWS to 25 amino acid residues spanning amino acids 632-656 at the C-terminus, and two NLSs of FLI1, NLS1 (63-90) in the N terminal domain and NLS2 (319-360) in the 3' ETS domain. All of the EWS-FLI1 fusion proteins completely reside in the nucleus using NLS2 of FLI1, suggesting that the manner of nuclear localization among variants of EWS-FLI1 is the same, and may not cause the different biological characteristics.

\section{Acknowledgements}

This work was supported in part by Grants-in-Aid for Scientific Research from the Ministry of Education, Science, Sports and Culture of Japan, and for Third-Term Comprehensive 10-Year Strategy for Cancer Control from the Ministry of Health, Labor and Welfare of Japan, and Health Sciences Research Grants from the Ministry of Health, Labor and Welfare of Japan.

\section{References}

1. Delattre O, Zucman J, Plougastel B, et al: Gene fusion with an ETS DNA-binding domain caused by chromosome translocation in human tumours. Nature 359: 162-165, 1992.

2. Kover H: Ewing tumor biology: perspective for innovative treatment approaches. Adv Exp Med Biol 532: 27-37, 2003

3. Arvand A and Denny CT: Biology of EWS/ETS fusions in Ewing's family tumors. Oncogene 20: 5747-5754, 2001.

4. Prasad DDK, Rao VN and Reddy ESP: Structure and expression of human FLI-1 gene. Cancer Res 52: 5833-5837, 1992.

5. Wasylyk B, Hahn SL and Giovane A: The Ets family of transcription factors. Eur J Biochem 211: 7-18, 1993.

6. Ohno T, Rao VR and Reddy SP: EWS/FLI-1 chimeric protein is a transcriptional activator. Cancer Res 53: 5859-5863, 1993.

7. May WA, Lessnick SL, Braun BS, et al: The Ewing's sarcoma $E W S / F L I-1$ fusion gene encodes a more potent transcriptional activator and is a more powerful transforming gene than FLI-1. Mol Cell Biol 13: 7393-7398, 1993.
8. Braun BS, Frieden R, Lessnick SL and May WA: Denny CT. Identification of target genes for the Ewing's sarcoma EWS/FLI fusion protein by representational difference analysis. Mol Cell Biol 15: 4623-4630, 1995.

9. Hahm KB, Cho K, Lee C, et al: Repression of the gene encoding the TGF- $\beta$ type II receptor is a major target of the EWS-FLI1 oncoprotein. Nat Genet 23: 222-227, 1999.

10. Zucman J, Melot T, Desmaze C, et al: Combinatorial generation of variable fusion proteins in the Ewing family of tumours. EMBO J 12: 4481-4487, 1993.

11. Zoubek A, Dockhorn B, Delattre O, et al: Does expression of different EWS chimeric transcripts define clinically distinct risk groups of Ewing tumor patients? J Clin Oncol 14: 1245-1251, 1996.

12. de Alava E, Kawai A, Healey JH, et al: EWS-FLI1 fusion transcript structure is an independent determinant of prognosis in Ewing's sarcoma. J Clin Oncol 16: 1248-1255, 1998.

13. Ozeki T, Paulussen M, Poremba C, et al: Genetic imbalances revealed by comparative genomic hybridization in Ewing tumors. Genes Chromosomes Cancer 32: 164-171, 2001.

14. Felsch JS, Lane WS and Peralta EG: Tyrosine kinase pyk2 mediates G-protein-coupled receptor regulation of the Ewing sarcoma RNA-binding protein EWS. Curr Biol 9: 485-488, 1999.

15. Belyanskaya LL, Gehrig PM and Gehring H: Exposure on cell surface and extensive arginine methylation of Ewing sarcoma (EWS) protein. J Biol Chemist 278: 18681-18687, 2001.

16. Bailly RA, Bosselut R, Zucman J, et al: DNA-binding and transcriptional activation properties of the EWS-FLI1 fusion protein resulting from the $\mathrm{t}(11 ; 22)$ translocation in Ewing sarcoma. Mol Cell Biol 14: 3230-3241, 1994.

17. Kim J, Lee K and Pelletier J: The DNA binding domains of the WT1 tumor suppressor gene product and chimeric EWS/WT1 oncoprotein are functionally distinct. Oncogene 16: 1021-1030, 1998.

18. Eguchi H, Ikuta T, Tachibana T, Yoneda Y and Kawajiri K: A nuclear localization signal of human aryl hydrocarbon receptor nuclear translocator/hypoxia-inducible factor 13 is a novel bipartite type recognized by the two components of nuclear pore-targeting complex. J Biol Chem 272: 17640-17647, 1997.

19. Ikuta T, Watanabe J and Kawajiri K: Characterization of the LxxLL motif in the aryl hydrocarbon receptor: effects on subcellular localization and transcriptional activity. J Biochem 131: 79-85, 2002

20. Ladanyi $\mathrm{M}$ and Gerald $\mathrm{W}$ : Fusion of the EWS and WT1 genes in the desmoplastic small round cell tumor. Cancer Res 54: 2837-2840, 1994.

21. Mattaj IW and Englmeier L: Nucleocytoplasmic transport: the soluble phase. Ann Rev Biochem 67: 265-306, 1998.

22. Nigg EA: Nucleocytoplasmic transport: signals, mechanisms and regulation. Nature 386: 779-787, 1997.

23. Boulukos KE, Pognonec P, Rabault B, Begue A and Ghysdael J: Definition of an Ets 1 protein domain required for nuclear localization in cells and DNA-binding activity in vitro. Mol Cell Biol 9: 5718-5721, 1989.

24. Lin PP, Brody RI, Hamelin AC, Bradner JE, Healey JH and Ladanyi M: Differential transactivation by alternative EWSFLI1 fusion proteins correlates with clinical heterogeneity in Ewing's sarcoma. Cancer Res 59: 1428-1432, 1999.

25. de Alava E, Panizo A, Antonescu CR, Pardo-Mindan J, Huvos AG, Barr FG and Ladanyi M: Association of EWS-FLI1 type 1 fusion with lower proliferative rate in Ewing's sarcoma. Am J Pathol 156: 849-855, 2000.

26. Bandres E, Malumbres R, Escalada A, et al: Gene expression profile of Ewing sarcoma cell lines differing in their EWS-FLI1 fusion type. J Pediatr Hematol Oncol 27: 537-542, 2005. 